\title{
Comparison of supercritical fluid extraction and thermal desorption methods for analysing organic compounds in house- dust
}

\author{
Papadopoulos A.* \\ Biomolecular Physics Laboratory, NCSR “Demokritos", Aghia Paraskevi, Greece \\ Received: 31/12/2020, Accepted: 26/09/2021, Available online: 22/01/2021 \\ *to whom all correspondence should be addressed: e-mail: thapap@rrp.demokritos.gr
}

https://doi.org/10.30955/gnj.003493

Graphical abstract
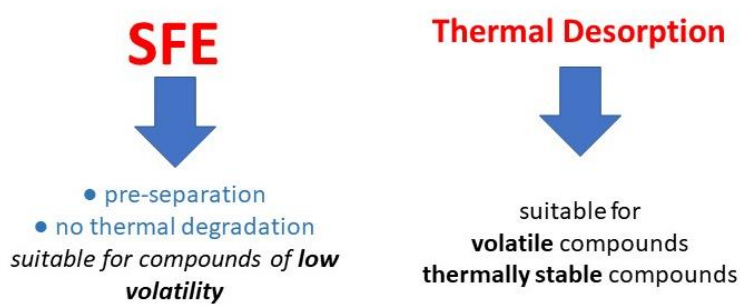

\begin{abstract}
This study has been focused on the comparison of the application of Thermal Desorption (TD) and Supercritical Fluid Extraction (SFE) methods for the identification and quantification of organic chemicals in house dust samples. To investigate how the results obtained by SFE and TD of house dust compare to one another and whether the SFE has advantages over the TD method, an aliquot of a house dust sample has been subjected to desorption at successively increasing temperatures. The thermal desorption unit used cryo - focusing on capillary tubing and was connected to a GC-MS combination. A quantity of the same house dust sample was extracted, using a method consisting of a two-step SFE with $\mathrm{CO}_{2}$ and $\mathrm{CO}_{2}+$ $5 \%$ of methanol, and GC-MS analysis of the eluates. The comparison of the results showed that the SFE method was superior to the TD for analysing indoor dust samples because of the pre-separation and the absence of thermal degradation, particularly for compounds of low volatility. However, TD could be more appropriate for relatively volatile or lower molecular weight range compounds and thermally stable compounds.
\end{abstract}

Keywords: $\mathrm{SFE}, \mathrm{CO}_{2}$, thermal desorption, thermal degradation, indoor dust.

\section{Introduction}

Numerous studies have demonstrated that indoor air (both gas and particle phases) contains a wide spectrum of organic pollutants in high concentrations (Ott et al.,
2006). Indoor dust is a fine heterogeneous mixture of organic and inorganic materials composed of skin cells, plant pollen, human and animal hairs, textile fibers, fungal spores, pollen, clay, as well as particulate matter emanating from carpet and furniture (Butte et al., 2002; Lioy et al., 2002). Indoor dust behaves as a repository and a concentrator of many organic contaminants and thus levels of contaminants in indoor dust can be used as a proxy to assess the exposure potential to contaminants in the indoor environment. Typically, carpeted surfaces are the largest reservoir of indoor dust. Furthermore, organic pollutants bound to dust are more persistent indoors due to a lack of biotic (e.g., microbial) and abiotic (e.g., photolysis) degradation and other dissipation processes (e.g., volatilization, dissolution) and consequently they have a greater exposure potential (Hwang et al., 2008). Household dust contains a complex mixture of hundreds of chemicals in varying concentrations based on the dust source. It has been identified as a major source of environmental contaminants including pesticides (Richards et al., 2016), polycyclic aromatic hydrocarbons (PAHs) (Galmiche et al., 2021), phthalates (Demirtepe et al., 2021), several metals (Hasanpour et al., 2020a), organic dyes (Hasanpour et al., 2020b) and other chemicals of human health concern (Roberts et al., 2009). Extraction of house dust can be used as a screening method for high boiling organic compounds in the indoor (Butte et al., 2002; Hilton et al., 2010; Papadopoulos et al., 2013). Epidemiological and clinical studies in the recent years have shown that the importance of the indoor air quality to human health should not be underestimated (US EPA, 2015). The assessment of indoor quality related to indoor dust requires efficient and reliable methods producing useful information (Lucattini et al., 2018; Melymuk et al., 2020).

Two different techniques are mainly employed to remove adsorbed species from the collected matrix; thermal extraction and solvent extraction. This study has been focused on the comparison of the application of Supercritical Fluid Extraction (SFE) and Thermal Desorption (TD) methods for the identification and 
quantification of other classes of low-volatile or nonvolatile organic compounds to which people may be exposed indoors. The use of supercritical $\mathrm{CO}_{2}$ as extraction fluid is advantageous because of its gas-like mobility and liquid-like solvating power. In addition, the properties of the fluid can easily be controlled by varying pressure and temperature. SFE tends to be more selective, faster and environmentally friendly than conventional extraction methods (Brunner et al., 2010; Papadopoulos, 2012). An alternative to the solvent extraction of indoor dust is thermal desorption (TD). Only minimal manipulation of the sample is required since no solvent or glassware is involved, eliminating the risk of external contamination. Among the advantages of the method are its rapidity and simplicity due to the direct approach. The extracted compounds are transferred online onto the analytical column of the GC. The introduction of a large part of the sample into the gas chromatograph decreases the limits of quantification, enhancing the sensitivity of the analysis. Drawbacks of the method are associated with the physical and chemical properties the detected compounds, such as volatility and thermal stability (Clement et al., 2000; Morawska et al., 2006). Thermal desorption followed by gas chromatography analysis was commonly used for the analysis of volatile organic compounds (VOCs) and SVOCs in indoor air (Barro et al., 2009; Garcia-Jares et al., 2009) but rarely used for the specific analysis of house dust and SVOCs in suspended particulate matter (Hirvonen et al., 1994; Pedersen et al., 2002).

In the present work two methods, SFE and thermal desorption, are applied with the intention of investigating the best approach to analyze house dust samples of a broad range of chemical classes, depending on the thermal stability and volatility of the extracted compounds. The investigation is focused on exploring the advantages of the one method over the other, considering selectivity, rapidity and minimal manipulation of the sample.

\section{Materials and methods}

\subsection{Sampling}

A house dust sample was collected from an apartment located in the city of loannina, in north-western Greece. The sample was taken in the living room, furnished with loose carpets. The larger part of the dust was sampled from the carpet surfaces. The flooring material in the living room was ceramic tiles. There was no open fireplace in the room. There were no pets in the apartment and no smoking was taking place on a regular basis.

The dust sample was collected_by means of the High Volume Small Surface Sampler (HVS3 Cascade Stack Sampling Systems) developed by U.S. EPA (Environmental Protection Agency) and manufactured by CS3 Inc, Sandpoint, ID, USA. The surface dust enters the system through the nozzle. The nozzle is specially designed to move across a floor with little resistance, while still maintaining a sufficient seal to collect a sample. The HVS3 sampling train is made from aluminium and has some Teflon tubing and gaskets and the catch bottle is made of
Teflon to avoid contamination with compounds associated with plastic materials. The samples were sieved for $5 \mathrm{~min}$ in a shaker, with a 100 mesh screen above the pan in order to remove particles larger than about $150 \mu \mathrm{m}$. The fine dust below $150 \mu \mathrm{m}$ passing through the sieve was weighed and preserved.

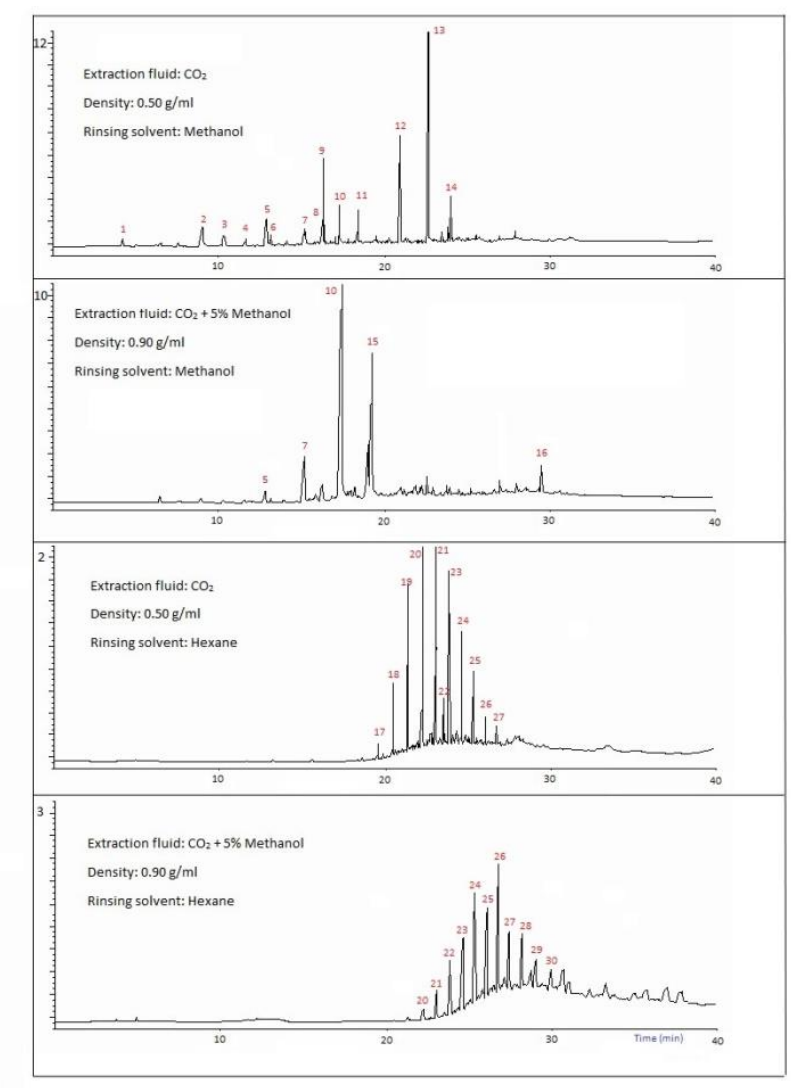

Figure 1. Chromatograms of two successive SFE of the same house dust sample and two rinses (methanol and hexane) of the ODS trap after each extraction. 1. Ethanol, 2-butoxy-; 2. Nonanoic acid; 3. Decanoic acid; 4. Undecanoic acid; 5. Dodecanoic acid; 6. 1,2-Benzenedicarboxylic acid, diethyl ester; 7. Tetradecanoic acid; 8. 1-Hexadecanol; 9. 1,2Benzenedicarboxylic acid, butyl 2-methylpropyl ester; 10. Hexadecanoic acid; 11. 1,2-Benzenedicarboxylic acid, dibutyl ester; 12. 1-Octadecanol; 13. 1,2-Benzenedicarboxylic acid, bis(2-ethylhexyl) ester; 14. Butyl benzyl phthalate; 15 .

Octadecanoic acid; 16. 5-cholestene-3-ol (3.beta.)-; 17. Docosane; 18. Tricosane; 19. Tetracosane; 20. Pentacosane; 21. Hexacosane; 22. Tetracosane, 2,6,10,15,19,23-hexamethyl-; 23. Heptacosane; 24. Octacosane; 25. Nonacosane; 26. Triacontane; 27. Hentriacontane; 28. Dotriacontane; 29. Tritriacontane; 30. Tetratriacontane.

\subsection{Supercritical fluid extraction (SFE)}

The extraction method followed is described in detail in Papadopoulos, 2012. The dust sample (0.5 g) was placed in a thimble, in the extraction chamber of the HP supercritical fluid extractor (HP 7680T) and was extracted with a combination of two steps (the first with $\mathrm{CO}_{2}$ of 0.5 $\mathrm{g} / \mathrm{ml}$ density and the second with $\mathrm{CO}_{2}$ of $0.9 \mathrm{~g} / \mathrm{ml}$ density with the addition of $5 \%$ of methanol). The chemicals used were: $\mathrm{CO}_{2}$ : Airgas SFE (Supercritical Fluid Extraction) 
Grade Carbon Dioxide, size 150 High Pressure Aluminum Cylinder, CGA-320. Methanol: Supelco anhydrous for analysis (max. 0.003\% $\mathrm{H}_{2} \mathrm{O}$ ) CAS \# 67-56-1. n-Hexane: Supelco for analysis CAS \# 110-54-3. Extracts were rinsed from an ODS collection trap (octadecyl chains bonded to silica spheres), with methanol and with n-hexane. Using that method, a very satisfactory selective pre-separation fractionation was achieved: The hydrophilic compounds were rinsed with methanol, whereas the lipophilic compounds were rinsed with $\mathrm{n}$-hexane. Compounds of smaller molecular weight were extracted during the first extraction step, and the extraction of heavier compounds occurred during the next step (higher $\mathrm{CO}_{2}$ density). An aliquot of each rinsing fraction was injected on-column to the gas chromatograph (HP 5890 Series II plus) equipped with mass spectrometry (HP 5972 series II). The corresponding chromatograms are presented in Figure 1. All chromatograms are normalized to the highest peak.

The lipophilic fractions (hexane) were analyzed by a nonpolar general-purpose column (OV1, Mega, Capillary columns laboratory, Via Plinio 29, Legnano MI, Italy) with a phase thickness of $0.1-0.15 \mu \mathrm{m}$, internal diameter 0.2 $\mathrm{mm}$ and $25 \mathrm{~m}$ length. The hydrophilic fractions (methanol) were analyzed by a Carbowax polar column (Mega-Acid, Mega, Capillary columns laboratory, Via Plinio 29, Legnano MI, Italy) with a phase thickness of $0.1-0.15 \mu \mathrm{m}$ and internal diameter $0.2 \mathrm{~mm}$. Helium was used as the GC carrier gas (Air Liquide, CAS \# 7440-59-7). The injection of extracts from samples was performed by hand. The injector temperature was maintained at $280^{\circ} \mathrm{C}$. The GC temperature programs were designed as given below: (a) OV1 column: $10^{\circ} \mathrm{C} / \mathrm{min}$ from 50 to $320^{\circ} \mathrm{C}$. (b) Mega-Acid column: $10^{\circ} \mathrm{C} / \mathrm{min}$ from 50 to $250^{\circ} \mathrm{C}$. Mass spectrometric detection in full scan mode was used to measure the Elspectra of the eluted compounds, carried out with the following parameters: (i) temperature of the transfer line at $300^{\circ} \mathrm{C}$, (ii) temperature of the ion source at $230^{\circ} \mathrm{C}$, (iii) temperature of the quadrupole filter at $150^{\circ} \mathrm{C}$ and (iv) mass range from $\mathrm{m} / \mathrm{z} 50$ to 550 . Data acquisition and processing were performed with a ChemStation data system (Agilent Technologies). As the main objective of the survey analysis was to qualitatively describe the organic content of the house dust and in view of the large number of compounds detected in the house dust extracts, a semi-quantitative analysis was deemed most suitable for classifying the compounds in three predefined concentration ranges: $(*)<20 \mu \mathrm{g} / \mathrm{g}$ dust, $(* *) 20$ $200 \mu \mathrm{g} / \mathrm{g}$ dust, $(* * *) \geq 200 \mu \mathrm{g} / \mathrm{g}$ dust.

\subsection{Thermal desorption (TD)}

Thermal desorption is the use of heat and a flow of inert gas to elute volatile compounds from solid or liquid matrices and transfer them to the analytical system which is invariably a gas Chromatograph (GC). If the extracted compounds are directly transferred onto the analytical column of the GC without re-concentration or re-focusing, the process is called "single stage" desorption. However, single stage desorption actually produces such broad component bands. This limitation is overcome by refocusing the desorbed volatiles in an adsorption / desorption stage before gas chromatographic separation. Refocusing is carried out by cryo-focusing on capillary tubing in front of the chromatographic column. This procedure reduces component bandwidths and improves the efficiency of the subsequent chromatographic separation.

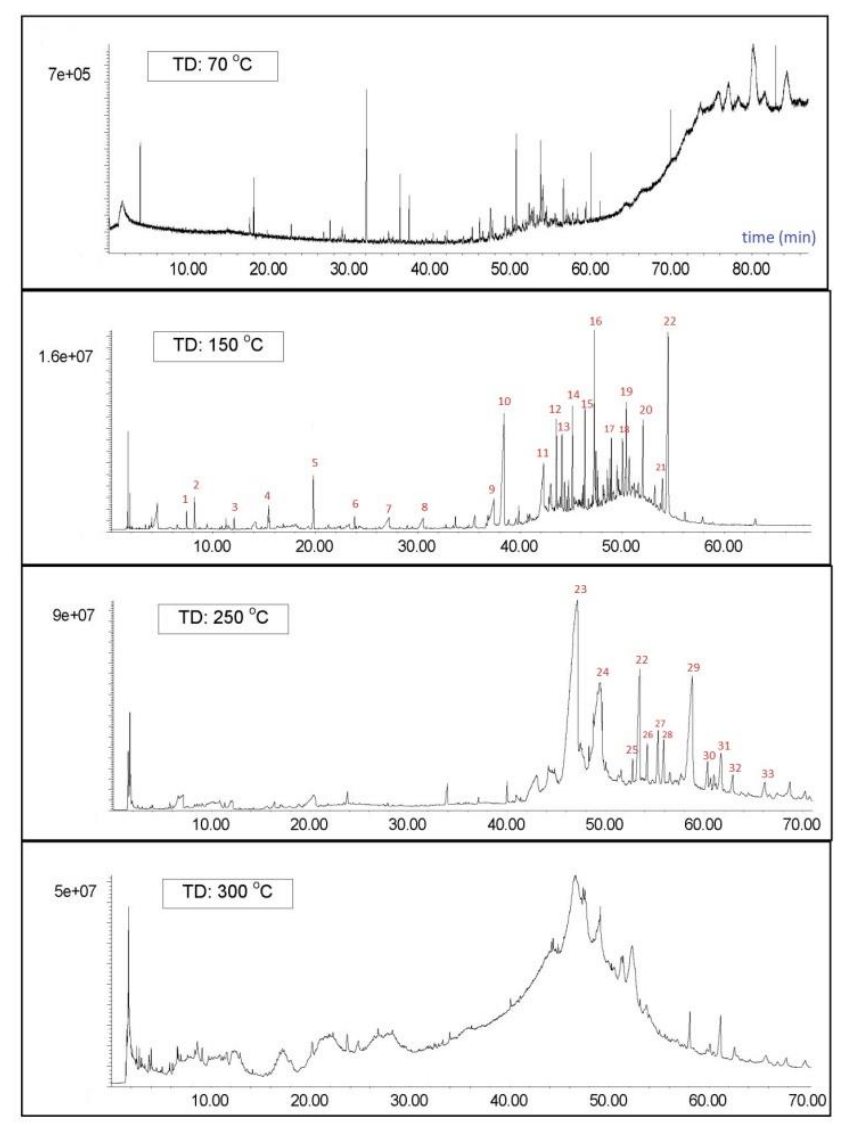

Figure 2. Chromatograms of successive thermal desorptions (at different temperatures) of the house dust sample. 1 . Hexanal; 2.

Octane; 3. Nonane; 4. Octanal; 5. Nonanal; 6. Decanal; 7. Nonanoic acid; 8. Decanoic acid; 9. Dodecanoic acid; 10 . Diethyl phthalate; 11. Tetradecanoic acid; 12. 1,2-Benzenedicarboxylic acid, bis(2-methylpropyl) ester; 13. 1-Tetradecanol; 14. 1,2Benzenedicarboxylic acid, dibutyl ester; 15. Eicosane; 16. 1-

Octadecanol; 17. Docosane; 18. Tricosane; 19. 1,2-

Benzenedicarboxylic acid, butyl phenylmethyl ester; 20 .

Tetracosane; 21. Pentacosane; 22. 1,2-Benzenedicarboxylic acid, bis(2-ethylhexyl) ester; 23. Hexadecanoic acid; 24. Octadecanoic acid; 25. Pentacosane; 26. Hexacosane; 27. Tetracosane, 2,6,10,15,19,23-hexamethyl-; 28. Heptacosane; 29. Octacosane; 30. Nonacosane; 31. Cholest-5-en-3-ol (3.beta.)-; 32. Triacontane; 33. Hentriacontane.

$5 \mathrm{mg}$ of the house dust sample has been subject to direct thermal desorption with the intention of comparing the composition of the eluates with that of the extracts obtained after extraction of the same sample using the SFE method.

The thermal desorption unit (thermal desorption cold trap injector/purge and trap injector, Chrompack, CP-4010 PTI/TCT) used cryo-focusing on capillary tubing and was connected to a GC-MS combination (HP 5890 series II plus - HP 5972 series II). 
A sample tube containing the dust sample was placed inside the desorption oven and connected to the fused silica capillary cold trap. In the next step desorption oven was heated, and the thermally desorbed components were cryo-focused on the cold trap, at a temperature of $2^{\circ} \mathrm{C}$. The capillary cold trap was flash-heated, and the components were injected into the analytical column (OV1, Mega, Capillary columns laboratory, Via Plinio 29, Legnano MI, Italy with a phase thickness of 0.1- $0.15 \mu \mathrm{m}$, internal diameter $0.2 \mathrm{~mm}$ and $25 \mathrm{~m}$ length), where they were separated.

Four different elution temperatures were used, eluting 5 $\mathrm{mg}$ of the house dust sample twice at each temperature:

1. Elutions $a \& b$ : at $70^{\circ} \mathrm{C}$ for $20 \mathrm{~min}$ (twice)

$\mathrm{GC}$ temperature program: $0-280^{\circ} \mathrm{C}, 4^{\circ} \mathrm{C} / \mathrm{min}$

2. Elutions $c \& d$ : at $150^{\circ} \mathrm{C}$ for $20 \mathrm{~min}$ (twice)

$\mathrm{GC}$ temperature program: $0-170^{\circ} \mathrm{C}, 4^{\circ} \mathrm{C} / \mathrm{min} \& 170-280^{\circ} \mathrm{C}$, $8^{\circ} \mathrm{C} / \min$

3. Elutions e \& $f$ : at $250^{\circ} \mathrm{C}$ for $20 \mathrm{~min}$ (twice)

$\mathrm{GC}$ temperature program: $0-170^{\circ} \mathrm{C}, 4^{\circ} \mathrm{C} / \mathrm{min} \& 170-300^{\circ} \mathrm{C}$, $8^{\circ} \mathrm{C} / \mathrm{min}$

\section{Elutions $g \& h$ : at $300^{\circ} \mathrm{C}$ for $20 \mathrm{~min}$ (twice)}

$\mathrm{GC}$ temperature program: $0-170^{\circ} \mathrm{C}, 4^{\circ} \mathrm{C} / \mathrm{min} \& 170-300^{\circ} \mathrm{C}$, $8^{\circ} \mathrm{C} / \min$

The parameters of the mass spectrometric detection were identical to those used for the SFE extracts. The same concentration ranges defined for the SFE extracts, have also been used for the TD results: $(*)<20 \mu \mathrm{g} / \mathrm{g}$ dust, $(* *)$ $20-200 \mu \mathrm{g} / \mathrm{g}$ dust, $(* * *) \geq 200 \mu \mathrm{g} / \mathrm{g}$ dust.

Figure 2 demonstrates the chromatograms of the first desorption at each temperature. All chromatograms are normalised to the highest peak. $70^{\circ} \mathrm{C}$ was chosen as the lower desorption temperature, since this is near to the maximum temperature occurring indoors, such as at the surface of radiators or on surfaces exposed to sunlight. However, at this temperature, all the peaks emerging in the chromatogram are of very low intensity, as shown in Figure 2. The concentration scale of the chromatograms for TD at $70^{\circ} \mathrm{C}$, is lower by at least a factor of 20 , compared to the scales of the chromatograms at higher TD temperatures, a fact that illustrates the very low quantities of the compounds desorbed at $70^{\circ} \mathrm{C}$.

The chromatogram with the highest number of peaks and giving the best resolution is obtained at a desorption temperature of $150^{\circ} \mathrm{C}$, (with the majority of the peaks eluting at $\mathrm{GC}$ column temperatures between $170^{\circ} \mathrm{C}$ and $\left.265^{\circ} \mathrm{C}\right)$.

At higher desorption temperatures, chromatograms become complex with incomplete $\left(250^{\circ} \mathrm{C}\right)$ or very little $\left(300^{\circ} \mathrm{C}\right)$ chromatographic resolution.

As mentioned above, the house dust sample was desorbed twice at each temperature. Comparison between the chromatograms obtained after the first and second desorption, at all temperatures, shows that the peaks corresponding to the second desorption are considerably lower than the equivalent peaks of the first desorption. There are no compounds desorbed during the second desorption which were not desorbed during the first one.

\section{Results and discussion}

\subsection{Comparison of thermally stable compounds}

The classes of compounds most commonly occurring in indoor dust were detected to be: fatty acids and some of their esters, n-alkanes, phthalates and alcohols. Other less frequently detected classes were other esters, phenols and aliphatic aldehydes (such as nonanal and decanal). In Table 1 are reported the semi-quantitative analytical results for compounds desorbed thermally and extracted from a house dust sample using SFE in concentrations higher than $20 \mu \mathrm{g} / \mathrm{g}$ dust (columns 6 and 7). Qualitative results of the GC-MS analysis of thermally desorbed compounds are reported in columns 2-5.

The detected compounds have been identified by MS library search. The identity was confirmed from the retention times at which compounds elute. The retention times were compared to those of reference compounds (when retention time reference values were available). In the other cases, the retention times were consistent with the estimated volatility of the compounds. The standard solutions used and the response factors applied for the semi-quantitative evaluation can be found in Papadopoulos, 2012.

The semi-quantitative data concerning the TD analysis correspond to the sum of all the desorption steps that have taken place.

During TD-GC-MS analysis, the whole amount of the desorbed compounds enters the analytical column. Using SFE of the house dust, and rinsing of the extracted compounds, four fractions of $1 \mathrm{ml}$ each were obtained for each sample. Only $0.5 \mathrm{ml}$ of each fraction, corresponding to $0.05 \%$ of the total extracted amount, were injected (on column) to the GC-MS.

Since the quantity of the house dust extracted by SFE is $500 \mathrm{mg}$, the amount of house dust corresponding to the portion of the extract injected for each analysis is $500 \mathrm{x}$ $0.05 / 100=0.25 \mathrm{mg}$ of house dust.

Therefore, if TD and SFE should have the same yields, thermal desorption of $0.25 \mathrm{mg}$ of house dust should result to comparable TIC signals as GC-MS analysis of the SFE samples.

However, for compounds with low concentrations, this quantity of house dust was often insufficient to give a TIC signal. Experience shows that in order to achieve reasonable GC-MS signals, $5 \mathrm{mg}$ of dust had to be thermally desorbed (20 times higher than the quantity corresponding to the portion of the SFE extract injected into GC-MS for analysis). 
Table 1. Qualitative analytical results of the TD analysis of house dust sample at increasing desorption temperatures, and semiquantitative analytical results of the compounds detected in highest concentration values in both SFE and TD analysis, of the same house dust sample. For the semi-quantitative evaluation, the concentration ranges were defined as follows: $(*)<20 \mu \mathrm{g} / \mathrm{g}$ dust, $(* *) 20-$ $200 \mu \mathrm{g} / \mathrm{g}$ dust, $(* * *) \geq 200 \mu \mathrm{g} / \mathrm{g}$ dust.

\begin{tabular}{|c|c|c|c|c|c|c|}
\hline \multirow[t]{2}{*}{ Identified compounds } & \multicolumn{3}{|c|}{ Desorption temperatures $\left({ }^{\circ} \mathrm{C}\right)$} & \multicolumn{3}{|c|}{ Semi-quantitation } \\
\hline & 70 & 150 & 250 & 300 & TD & SFE \\
\hline Tetracosane & & $\checkmark$ & & & $* *$ & $* *$ \\
\hline Pentacosane & & $\checkmark$ & $\checkmark$ & & $* *$ & $* *$ \\
\hline Hexacosane & & $\checkmark$ & $\checkmark$ & & $* *$ & $* *$ \\
\hline Heptacosane & & & $\checkmark$ & & $* *$ & $* *$ \\
\hline Octacosane & & & $\checkmark$ & & * & $* *$ \\
\hline Nonacosane & & & $\checkmark$ & & $* *$ & $* *$ \\
\hline Tetracosane, 2,6,10,15,19,23-hexamethyl- & & & $\checkmark$ & & $* *$ & $* *$ \\
\hline Triacontane & & & $\checkmark$ & & $* *$ & $*$ \\
\hline Hentriacontane & & & $\checkmark$ & & $* *$ & $* *$ \\
\hline Dotriacontane & & & $\checkmark$ & & $*$ & $*$ \\
\hline \multicolumn{7}{|l|}{$2,6,10,14,18,22-$ Tetracosahexaene, $2,6,10,15$} \\
\hline 19,23-hexamethyl-, (all-E)- (Squalene) & & $\checkmark$ & & & $* * *$ & $* * *$ \\
\hline Dodecanoic acid & & $\checkmark$ & & & $*$ & $* *$ \\
\hline Tetradecanoic acid & & $\checkmark$ & $\checkmark$ & & $* *$ & $* * *$ \\
\hline Pentadecanoic acid & & & $\checkmark$ & & $*$ & $* *$ \\
\hline Hexadecanoic acid & & & $\checkmark$ & & $* * *$ & $* * *$ \\
\hline Heptadecanoic acid & & & $\checkmark$ & & $*$ & $* *$ \\
\hline Octadecanoic acid & & & & & $*$ & $* *$ \\
\hline 9-Hexadecenoic acid & & & & & $*$ & $* *$ \\
\hline 9-Octadecenoic acid & & & $\checkmark$ & & $*$ & $* * *$ \\
\hline Hexadecanoic acid, methyl ester & & $\checkmark$ & & & $* *$ & $* *$ \\
\hline Hexadecanoic acid, tetradecyl ester & & & $\checkmark$ & $\checkmark$ & $* *$ & $*$ \\
\hline Hexadecanoic acid, hexadecyl ester & & & & & * & ** \\
\hline 9-Hexadecenoic acid, octadecyl ester, (Z)- & $\checkmark$ & & $\checkmark$ & & $*$ & $* *$ \\
\hline 1,2-Benzenedicarboxylic acid, dibutyl ester & & $\checkmark$ & & & * & $* *$ \\
\hline 1,2-Benzenedicarboxylic acid, bis(2-ethylhexyl) ester & & $\checkmark$ & $\checkmark$ & & $* * *$ & $* * *$ \\
\hline 1-Dodecanol & & & $\checkmark$ & & $* *$ & $*$ \\
\hline 1-Hexadecanol & & $\checkmark$ & $\checkmark$ & & $*$ & $* *$ \\
\hline 1-Octadecanol & & $\checkmark$ & & & $* *$ & $*$ \\
\hline Phenol, 4-(1,1,3,3-tetramethylbutyl)- & & $\checkmark$ & & & * & $* *$ \\
\hline Cholest-5-en-3-ol (3.beta.)- & & $\checkmark$ & & & $* *$ & $* *$ \\
\hline
\end{tabular}

Several factors have to be taken into consideration for the comparison of TD and SFE results:

1. The formation of thermal degradation products during thermal desorption.

2. The higher sensitivity of the TD analysis, because of the larger amount of house dust desorbed or extracted per analysis, as explained above.

3. The incomplete recovery by TD of higher boiling polar and thermally labile compounds.

4. The incomplete chromatographic resolution of the eluates at higher desorption temperatures during TD.

5. The lower starting temperature of the chromatographic programme for the TD eluates, which is at $0^{\circ} \mathrm{C}$, compared to that used for the SFE extracts, where the starting temperature is $50^{\circ} \mathrm{C}$.

The detection of a number of thermally stable compounds, such as C15 - C23 n-alkanes, only by TD and not by SFE, may be due to the higher sensitivity of the TD analysis, because of the larger amount of house dust desorbed or extracted per analysis.

However, supercritical fluid extraction of an amount of house dust larger than $500 \mathrm{mg}$, might result in detection of these lower boiling point compounds, even by using the SFE method.

$\mathrm{C}_{33}$ and $\mathrm{C}_{34} \mathrm{n}$-alkanes were only detected in the SFE extract and not after TD analysis of the house dust. This is probably due to the incomplete desorption of tritriacontane and tetratriacontane, the highest boiling point $\mathrm{n}$-alkanes detected.

Saturated fatty acids, have been more completely recovered by SFE than by TD. Several have been detected at a higher concentration range and a few branched fatty acids of high boiling points, were only extracted by SFE and not by TD. 
Table 2. Qualitative analytical results of the TD analysis of the house dust at increasing desorption temperatures, for compounds detected only by TD and not by SFE analysis

Identified compounds

\begin{tabular}{|c|c|c|c|}
\hline & 150 & 250 & 300 \\
\hline Acetaldehyde & & $\checkmark$ & \\
\hline Propanal & & & $\checkmark$ \\
\hline Propanal, 2-methyl- & & $\checkmark$ & $\checkmark$ \\
\hline 2-Butanone & & $\checkmark$ & \\
\hline Furan, 2-methyl- & & $\checkmark$ & $\checkmark$ \\
\hline Furan, 3-methyl- & & $\checkmark$ & $\checkmark$ \\
\hline Furan, tetrahydro- & & $\checkmark$ & $\checkmark$ \\
\hline 1-Propanol, 2-methyl- & $\checkmark$ & & \\
\hline Pentanal & & $\checkmark$ & \\
\hline Butanal, 3-methyl- & $\checkmark$ & $\checkmark$ & $\checkmark$ \\
\hline Butanal, 2-methyl- & & & $\checkmark$ \\
\hline Benzene & & $\checkmark$ & \\
\hline 1-Butanol & $\checkmark$ & $\checkmark$ & \\
\hline Acetic acid & $\checkmark$ & $\checkmark$ & \\
\hline Pyridine & & $\checkmark$ & $\checkmark$ \\
\hline 1H-Pyrrole & & $\checkmark$ & $\checkmark$ \\
\hline 2-Propanone, 1-hydroxy- & & & $\checkmark$ \\
\hline Benzene, methyl- & & & $\checkmark$ \\
\hline 2-Furancarboxaldehyde & & $\checkmark$ & $\checkmark$ \\
\hline Pentanenitrile, 4-methyl- & & $\checkmark$ & \\
\hline 1H-Pyrrole, 2-methyl- & & $\checkmark$ & \\
\hline 2-Furanmethanol & $\checkmark$ & $\checkmark$ & \\
\hline Pyrido[2,3-d]pyrimidine & & $\checkmark$ & \\
\hline Pyrazine, 2,5-dimethyl- & & $\checkmark$ & \\
\hline 1H-Pyrrole, 2,4-dimethyl- & & $\checkmark$ & \\
\hline $2(5 \mathrm{H})$ - Furanone & & $\checkmark$ & $\checkmark$ \\
\hline Pyridine, 2,4-dimethyl- & & & $\checkmark$ \\
\hline Benzaldehyde & & $\checkmark$ & \\
\hline 2-Furancarboxaldehyde, 5-methyl- & & $\checkmark$ & $\checkmark$ \\
\hline Trisulfide, dimethyl & & $\checkmark$ & \\
\hline 2(5H)-Furanone, 3-methyl- & & $\checkmark$ & \\
\hline Furan, 2-pentyl- & $\checkmark$ & & \\
\hline 1,3-Cyclopentanedione & & $\checkmark$ & $\checkmark$ \\
\hline Pyridine, 2-ethyl-6-methyl & & & $\checkmark$ \\
\hline 3-ethyl-4-methyl-2-pyrazoline & & $\checkmark$ & \\
\hline Ethanone, 1-phenyl- & & $\checkmark$ & \\
\hline 4(1H)-Pyridinone, 2,3-dihydro-1-methyl- & & $\checkmark$ & \\
\hline Pyrazine, 2-ethyl-3,5-dimethyl- & & $\checkmark$ & \\
\hline Phenol, 2-methoxy- & & $\checkmark$ & \\
\hline Benzene, 1-ethoxy-4-methyl- & & $\checkmark$ & \\
\hline 1H-Pyrrole-2-acetonitrile, 1-methyl- & & & $\checkmark$ \\
\hline 4H-Pyran-4-one, 3-hydroxy-2-methyl- & & $\checkmark$ & $\checkmark$ \\
\hline Pyrazine, 2-methyl-5-(1-propenyl)-, (E)- & & $\checkmark$ & $\checkmark$ \\
\hline 3,5,6-trimethyl-4H-1,2,4-dithiazine & & $\checkmark$ & \\
\hline 1-Dodecanethiol & & $\checkmark$ & $\checkmark$ \\
\hline 4-Toluenesulfonamide & $\checkmark$ & & \\
\hline Ethanol, 2-(dodecyloxy)- & $\checkmark$ & $\checkmark$ & \\
\hline Ethanone, 2,2-dimethoxy-1,2-diphenyl- & $\checkmark$ & & \\
\hline N-Ethyl-N-methyl-4-phenetidine & $\checkmark$ & & \\
\hline 11,12-Dihydrobenzo[b]fluoranthene & $\checkmark$ & & \\
\hline 2,4-dioctylphenol & $\checkmark$ & & \\
\hline 1H-Purin-6-amine, [(2-fluorophenyl)methyl]- & $\checkmark$ & & \\
\hline Hexadecanamide & $\checkmark$ & $\checkmark$ & $\checkmark$ \\
\hline 9-Octadecenamide, (Z)- & & $\checkmark$ & \\
\hline Octadecanamide & & $\checkmark$ & \\
\hline Cholesta-4,6-dien-3-ol, (3.beta.)- & & $\checkmark$ & \\
\hline 5-Cholestene-3-ol (3.beta.)-, propanoate & & $\checkmark$ & \\
\hline 5-Cholestene-3-ol (3.beta.)-, carbonochloridate & & $\checkmark$ & $\checkmark$ \\
\hline
\end{tabular}


For most of these compounds, quantitative thermal desorption, if ever achieved, would need long desorption times. Due to the incomplete chromatographic resolution of the eluates at higher desorption temperatures during TD, some of the compounds that may have been desorbed at $250^{\circ} \mathrm{C}$, could be hidden in the unresolved background (hump) of the chromatogram.

Due to the higher starting temperature of the chromatographic program for the SFE extracts $\left(50^{\circ} \mathrm{C}\right)$ compared to that used for the TD eluates $\left(0^{\circ} \mathrm{C}\right)$, volatile compounds such as hexanal, wouldn't have been detected by using the SFE method, even if they were extracted.

\subsection{Thermal degradation products}

A large number of compounds was detected only after thermal desorption of the house dust and not with extraction by SFE. In Table 2 these compounds are reported in elution order.

Although the majority of the compounds have low boiling points, they were desorbed at high temperatures, which leads to the assumption that they are more likely to be produced by thermal degradation of compounds of biological origin, due to the high desorption temperatures, rather than contained in house dust and not extracted by SFE.

The presence of furans in the TD extracts supports the above hypothesis, since the most important reaction pathway for furan formation involves the interaction of carbohydrates with amino acids (Rogge et al., 1991), which are the main components of biological material and likely to react at elevated temperatures used during thermal desorption.

Unsaturated fatty acids thermally decompose to release aldehydes at quite low temperatures, something that could explain the frequent detection of aldehydes (especially of low boiling points, at high desorption temperatures) during TD.

\section{Conclusions}

The proposed SFE procedure is superior for the identification and quantification of organic chemicals in house dust because of:

\section{- pre-separation and}

- absence of thermal degradation

SFE is particularly suitable for compounds of low volatility. For the analysis of more volatile compounds, the chromatographic separation has to start at a lower temperature and larger amounts of house dust have to be extracted.

TD may be appropriate for:

- relatively volatile or lower molecular weight range compounds

\section{- thermally stable compounds}

In practice, thermal desorption of house dust is limited to temperatures not much higher than $150^{\circ} \mathrm{C}$. In higher desorption temperatures, chromatographic resolution becomes insufficient for separating desorbed compounds. In part, this may be due to lacking pre-separation of the desorbed compounds and in part, it appears to be caused by thermal decomposition (probably of biological material).

It has not been within the scope of this work to analyze whether and in how far thermal desorption of house dust may be used to provide, through the analysis of thermal decomposition products, information on the biological material contained in it.

\section{Acknowledgements}

We acknowledge support of this work by the project "NCSRD INRASTES research activities in the framework of the national RIS3." (MIS 5002559) which is implemented under the "Action for the Strategic Development on the Research and Technological Sector", funded by the Operational Programme "Competitiveness, Entrepreneurship and Innovation" (NSRF 2014-2020) and co-financed by Greece and the European Union (European Regional Development Fund).

\section{References}

Barro R., Regueiro J., Llompart M. and Garcia-Jares C. (2009), Analysis of industrial contaminants in indoor air: Part 1. Volatile organic compounds, carbonyl compounds, polycyclic aromatic hydrocarbons and polychlorinated biphenyls. Journal of Chromatography A, 1216(3), 540-566.

Brunner G. (2010), Applications of Supercritical Fluids. Annual Review of Chemical and Biomolecular Engineering, 1, 321342.

Butte W. and Heinzow B. (2002), Pollutants in house dust as indicators of indoor contamination. Reviews of Environmental Contamination and Toxicology, 175, 1-46.

Clement M., Arzel S., Le Bot B., Seux R. and Millet M. (2000), Adsorption/thermal desorption-GC/MS for the analysis of pesticides in the atmosphere. Chemosphere, 40(1), 49-56.

Demirtepe H., Melymuk L., Codling G., Murínová L.P., Richterová D., Rašplová V., Trnovec T. and Klánová J. (2021), Targeted and suspect screening of plasticizers in house dust to assess cumulative human exposure risk. Science of The Total Environment, 781, 146667

Galmiche M., Delhomme O., François Y-N. and Millet M. (2021), Environmental analysis of polar and non-polar Polycyclic Aromatic Compounds in airborne particulate matter, settled dust and soot: Partl: Sampling and sample preparation. Trends in Analytical Chemistry, 134, 116146

Garcia-Jares C., Regueiro J., Barro R., Dagnac T. and Llompart M. (2009), Analysis of industrial contaminants in indoor air. Part 2. Emergent contaminants and pesticides. Journal of Chromatography A, 1216(3), 567-597.

Hasanpour M. and Hatami M. (2020), Application of threedimensional porous aerogels as adsorbent for removal of heavy metal ions from water/wastewater: A review study. Advances in Colloid and Interface Science, 284, 102247

Hasanpour M. and Hatami M. (2020), Photocatalytic performance of aerogels for organic dyes removal from wastewaters: Review study. Journal of Molecular Liquids, 309, 113094

Hilton D.C., Jones R.S., and Sjodin A. (2010), A method for rapid, non-targeted screening for environmental contaminants in 
household dust. Journal of Chromatography A, 1217(44), 6851-6856.

Hirvonen A., Pasanen P., Tarhanen J., Ruuskanen J. (1994), Thermal Desorption of Organic Compounds associated with Settled Household Dust. Indoor Air, 4, 255-264.

Hwang, H-M, Park, E-K, Young T.M. and Hammock B.D. (2008), Occurrence of endocrine-disrupting chemicals in indoor dust. Science of the Total Environment, 404(1), 26-35.

Lioy P.J., Freeman N.C.G. and Millette J.R. (2002), Dust: A metric for use in residential and building exposure assessment and source characterization. Environmental Health Perspectives, 110(10), 969-983.

Lucattini L., Poma G., Covaci A., de Boer J., Lamoree M.H. and Leonards P.E.G. (2018). A review of semi-volatile organic compounds (SVOCS) in the indoor environment: occurrence in consumer products, indoor air and dust. Chemosphere, 201, 466-482.

Melymuk L., Demirtepe H. and Jílková S.R. (2020), Indoor dust and associated chemical exposures. Current Opinion in Environmental Science \& Health, 15, 1-6.

Morawska L. and Salthammer T. (2006), Fundamentals of Indoor Particles and Settled Dust, in Indoor Environment. Wiley-VCH Verlag GmbH \& Co. KGaA, 1-46.

Ott W.R., Steinemann A.C. and Wallace L.A. (2006), Exposure Analysis. Taylor \& Francis.

Papadopoulos A. (2012), Optimization of variables on the supercritical fluid extraction of high-boiling organic compounds in house-dust. Journal of Supercritical Fluids, 62, 123-134.

Papadopoulos A., Vlachogiannis D., Maggos T., Sfetsos A. and Karayiannis M.I. (2013), A semi-quantitative approach for analysing low-volatile organic compounds in house dust using an SFE method: Significant common features and particular differences of the extracts. The Journal of Supercritical Fluids, 82, 268-281.

Pedersen E.K., Bjørseth O., Syversen T., Mathiesen M. (2002), Emissions from heated indoor dust. Environment International, 27(7), 579-87.

Richards J., Reif R., Luo Y., Gan J. (2016), Distribution of pesticides in dust particles in urban environments. Environmental Pollution, 214, 290-298

Roberts J.W., Wallace L.A., Camann D.E., Dickey P., Gilbert S.G., Lewis R.G., Takaro T.K. (2009), Monitoring and Reducing Exposure of Infants to Pollutants in House Dust. Reviews of Environmental Contamination and Toxicology, 201, 1-39.

Rogge W.F., Hildemann L.M., Mazurek M.A., Cass G.R., and Simoneit B.R.T. (1991), Sources of fine organic aerosol .1. Charbroilers and meat cooking operations. Environmental Science \& Technology, 25(6), 1112-1125.

US EPA O. (2015), Indoor Air Quality by Building Type [WWW Document]. US EPA. URL. https://www.epa.gov/indoor-airquality-iaq/indoor-air-quality-buildingtype. 